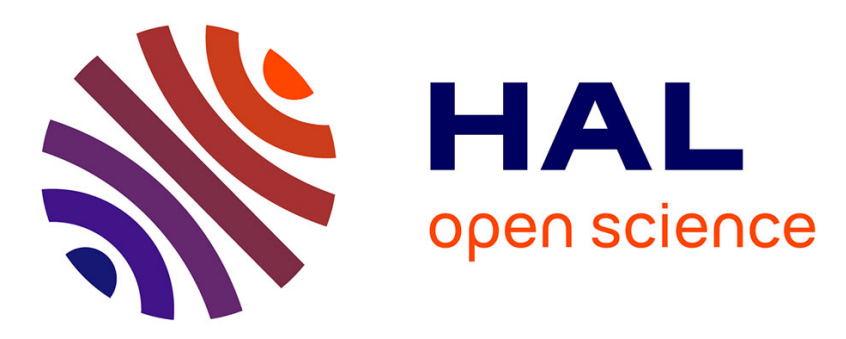

\title{
Numerical Study of Railway Track Dynamics: Case of a Transition Zone
}

Elodie Arlaud, Sofia Costa d'Aguar, Etienne Balmès, Guillaume Faussurier

\section{To cite this version:}

Elodie Arlaud, Sofia Costa d'Aguar, Etienne Balmès, Guillaume Faussurier. Numerical Study of Railway Track Dynamics: Case of a Transition Zone. The Third International Conference on Railway Technology: Research, Development and Maintenance, Apr 2016, Cagliari, Italy. pp.1-20. hal01341177

\section{HAL Id: hal-01341177 \\ https://hal.science/hal-01341177}

Submitted on 4 Jul 2016

HAL is a multi-disciplinary open access archive for the deposit and dissemination of scientific research documents, whether they are published or not. The documents may come from teaching and research institutions in France or abroad, or from public or private research centers.
L'archive ouverte pluridisciplinaire HAL, est destinée au dépôt et à la diffusion de documents scientifiques de niveau recherche, publiés ou non, émanant des établissements d'enseignement et de recherche français ou étrangers, des laboratoires publics ou privés. 


\title{
Numerical Study of Railway Track Dynamics: Case of a Transition Zone
}

\author{
January 5, 2016 \\ E. Arlaud ${ }^{12}$, S. Costa D’ Aguiar ${ }^{1}$, E. Balmes ${ }^{2}{ }^{3}$, G. Faussurier ${ }^{2}$
}

\begin{abstract}
The main objective of this paper is to present an innovative numerical tool to represent the track and platform dynamic behavior under passing trains. Dynavoie is currently being developed for engineering purposes at SNCF. It is specifically designed to correctly reproduce the track and platform dynamic behaviors in the time domain. This model is based on the Finite Element Method (FEM), adapted to the railway track using model reduction techniques. Taking advantage of the periodicity of the track introduced by the regular sleeper spacing, one or several basic slices containing all the geometric properties of the structure can be identified. Static and periodic responses of these slices are computed. Then, reduction is performed using these deformations.

\footnotetext{
${ }^{1}$ SNCF, Innovation \& Research, 40 avenue des Terroirs de France, 75611, Paris Cedex 12, France

${ }^{2}$ Arts et Métiers ParisTech, Laboratoire PIMM, CNRS UMR 8006 151, boulevard de l'hôpital, 75013, Paris, France

${ }^{3}$ SDTools 44, Rue Vergniaud, 75013, Paris, France
} 
Thus the number of degrees of freedom of the model is highly reduced. This reduction induces a decrease in computation time with limited accuracy loss in the representation of track dynamics. The methodology will be further described in this paper. The second novelty of this work is to use this model to analyze the dynamic behavior of a transition zone located in a French high speed line.

Keywords: Railway track, modeling, dynamic, transition zone, High Speed Line.

\section{Introduction}

The French High Speed line network has developed for more than 30 years mainly on analytical and semi-empirical basis regarding track and platform conception. Currently, more than $30000 \mathrm{~km}$ of lines are operated in France among which $2000 \mathrm{~km}$ of HS lines. The French railway network can be seen as a laboratory to experiment new track designs: under sleeper pads (USP), soft rail pads, bituminous layer [1]... Otherwise to assess the mechanical performance of the infrastructure from the dynamic point of view, numerical models are of great interest [2]. In addition to feedback, models are also useful in areas presenting unexpected behavior (such as ballast layer dynamic instability, hanging sleepers, differential settlement,etc.) in order to diagnose the track and to recommend adapted solutions . To design specific solution $[3,4]$ or to assess the dynamic behavior of tracks, many models have been proposed [5, 6, 7, 8, 9]. A first category among the cited models [6, 8], uses a full 3D Finite Element (FE) model of track. Geometry of the track can then be correctly modeled, taking into account changes in materials or geometric discontinuities, but time computations can reach several days of calculation as reported in both papers. A second category of models $[5,7,9]$, are based on a 2.5D FEM/BEM modeling of the track. Calculations are made on an uniform section of the track which is considered as invariant in the longitudinal direction. This hypothesis allows to highly reducing the memory required by modeling, but implies difficulties to represent geometric discontinuities or longitudinal change in material properties.

The model used in this paper, Dynavoie, is a hybrid approach between these two categories. The modeling characteristics are close to the ones proposed by [10] who models track as composed of periodic cells and perform a Floquet transform in the basic cell. An additional reduction step is proposed in this paper.

Dynavoie is currently being developed for engineering purposes at SNCF. This numerical model is specifically designed to correctly reproduce the track and platform dynamic behaviors in the time domain. This model is based on the Finite Element Method (FEM) plus model reduction techniques that are used to reduce the number of degrees of freedom. This reduction decreases computation time and memory requirements with limited accuracy loss in the representation of track dynamics.

Dynavoie methodology is described in this paper. Then, a specific area of the French railway network is chosen. First, a brief analysis based on experimental mea- 
surements is proposed. Then Dynavoie is used to model this area and provides further understanding of the track behavior.

\section{Dynavoie Method}

Numerical simulation of railway tracks by usual Finite Element Method can be time and resource consuming. Dynavoie aims at proposing a simulation of railway track dynamics in a short amount of time and of storage required by using Ritz reduction based on a substructuring technique. The method used in this work is based on the geometric pseudo-periodicity of the track. In the context of model reduction, the challenge is to consider pertinent deformations to chose accurate reduced Degrees of Freedom (DOFs).The following sections detail these choices and the corresponding calculation steps.

\subsection{Reduction method}

Railway track can be considered as periodic following sleepers. Every slice includes one sleeper, so spatial periodicity is equal to sleeper spacing, $\Delta x=60 \mathrm{~cm}$ in France. The global track can be generated by translations following $x$ of the basic slice.

On a slice $N$, displacements at the degrees of freedom of the mesh $\left\{\mathbf{q}^{\mathbf{N}}\right\}$ are decomposed as following:

$$
\left\{\mathbf{q}^{\mathbf{N}}\right\}=\left\{\begin{array}{llll}
q_{l}^{N} & q_{c}^{N} & q_{\text {rail }}^{N} & q_{r}^{N}
\end{array}\right\}^{T}
$$

$q_{l}^{N}$ are DOFs of the left interface of the slice $N, q_{r}^{N}$ are the DOFs of the right interface, $q_{r a i l}^{N}$ are DOFs corresponding to the top of the rail (see Figure 2) and $q_{c}^{N}$ are the DOFs corresponding to the center of the slice (all the DOFs that are not in other categories).

This separation of DOFs is useful to ensure that solution found using the reduction basis is kinematically admissible (continuity of displacement between adjacent slices).

First step is to find a reduction basis $[\mathbf{T}]$ giving

$$
\{\mathbf{q}\} \simeq[\mathbf{T}]\left\{\mathbf{q}_{\mathbf{R}}\right\},
$$

with $\{\mathbf{q}\}=\left\{\begin{array}{lll}q^{1} & \cdots & q^{N}\end{array}\right\}$ and $\left\{\mathbf{q}_{\mathbf{R}}\right\}=\left\{\begin{array}{lll}q_{R}^{1} & \cdots & q_{R}^{N}\end{array}\right\},\left\{\mathbf{q}_{\mathbf{R}}\right\}$ being the displacement in the reduced model.

A representation of matrix $[\mathbf{T}]$ is proposed in Figure 1 for a model of three slices. Colored blocks stand for non-zero terms and colored points for DOFs.

This matrix has been indexed by block, following:

- Columns are the number of the slice considered

- Line 1 (red) represents slice 1 without right its interface.

- Line 2 (blue) represents slice 1 right interface and slice 2 left interface. 


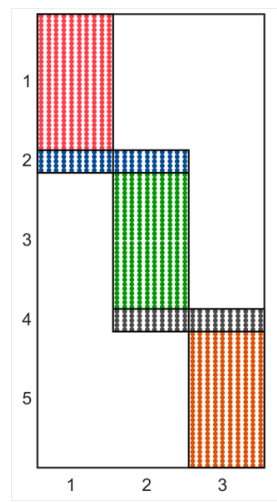

Figure 1: Representation of the reduction matrix $[\mathbf{T}]$

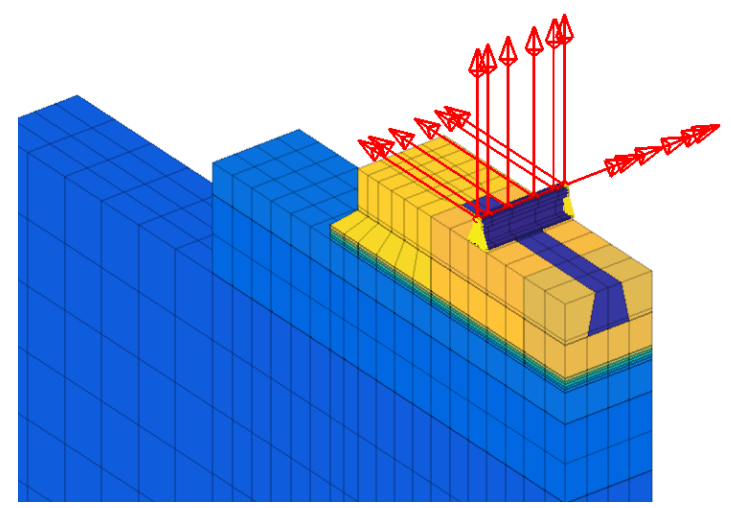

Figure 2: Degrees of freedom corresponding to the top of the rail

- Line 3 (green) represents slice 2 without its interfaces left and right.

- Line 4 (gray) represents slice 2 right interface and slice 3 left interface.

- Line 5 (orange) is the third and last slice without its left interface.

One of the key principles of Ritz reduction is that if the solution belongs to the considered subspace, then it will be exactly recovered solving the reduced problem. Two meaningful families of solutions in dynamic study of railway tracks under passing trains are static response under fix loading and wave propagation along track $[11,12$, 13].

This is why periodic solutions to these two problems will be used to build the reduction basis.

\subsection{Periodic solutions}

The conventions used in this work regarding Fourier transform are fully described in [14]. 
Knowing the Fourier transform $U$ of a real-valued field, $u$ is recovered computing the inverse Fourier transform given by

$$
\begin{array}{r}
u(n \Delta x)=\frac{1}{2 \pi} \int_{0}^{\pi} 2\left(\operatorname{Re}\left(U\left(\kappa_{c x}\right)\right) \cos \left(n \kappa_{c x}\right)\right. \\
\left.-\operatorname{Im}\left(U\left(\kappa_{c x}\right)\right) \sin \left(n \kappa_{c x}\right)\right) d \kappa_{c x}
\end{array}
$$

Using Equation (3) displacement of the periodic track structure with $\mathrm{N}$ slices can be written as:

$$
\{\mathbf{q}(\mathbf{x})\}=\left\{\begin{array}{c}
q^{1}(\Delta x) \\
q^{2}(2 \Delta x) \\
\vdots \\
q^{N}(N \Delta x)
\end{array}\right\}=\left\{\begin{array}{c}
\int_{0}^{\pi}\left(\operatorname{Re}\left(Q_{\kappa_{c x}(\Delta x)}\right) \cos \left(\kappa_{c x}\right)-\operatorname{Im}\left(Q_{\kappa_{c x}}(\Delta x)\right) \sin \left(\kappa_{c x}\right)\right) \mathrm{d} \kappa_{c x} \\
\int_{0}^{\pi}\left(\operatorname{Re}\left(Q_{\kappa_{c x}}(\Delta x)\right) \cos \left(2 \kappa_{c x}\right)-\operatorname{Im}\left(Q_{\kappa_{c x}}(\Delta x)\right) \sin \left(2 \kappa_{c x}\right)\right) \mathrm{d} \kappa_{c x} \\
\vdots \\
\int_{0}^{\pi}\left(\operatorname{Re}\left(Q_{\kappa_{c x}}(\Delta x)\right) \cos \left(N \kappa_{c x}\right)-\operatorname{Im}\left(Q_{\kappa_{c x}}(\Delta x)\right) \sin \left(N \kappa_{c x}\right)\right) \mathrm{d} \kappa_{c x}
\end{array}\right\}
$$

Approximation of this integral is made by discretization on wavenumbers, taking evenly spaced values of $\kappa_{c x}$ in the $[0 \pi]$ interval, so $\kappa_{c x}=2 \pi / n$ with $n \in[0 N]$. So Equation (4) can be written

$$
\{\mathbf{q}(\mathbf{x})\}_{N \times 1}=[\mathbf{E}]_{N \times 2 N}\left\{\mathbf{Q}_{\mathbf{0}}\right\}_{2 N \times 1}
$$

with:

$$
\begin{aligned}
& \text { with: } \\
& {[\mathbf{E}]_{N \times 2 N}=\left\{\begin{array}{ccccc}
\cos (1 \cdot 2 \pi) & -\sin (1 \cdot 2 \pi) & \ldots & \cos \left(\frac{1 \cdot 2 \pi}{N}\right) & -\sin \left(\frac{1 \cdot 2 \pi}{N}\right) \\
\cos (2 \cdot 2 \pi) & -\sin (2 \cdot 2 \pi) & \ldots & \cos \left(\frac{2 \cdot 2 \pi}{N}\right) & \sin \left(\frac{2 \cdot 2 \pi}{N}\right) \\
\vdots & \vdots & \ldots & \vdots & \vdots \\
\cos (N \cdot 2 \pi) & -\sin (N \cdot 2 \pi) & \ldots & \cos \left(\frac{N \cdot 2 \pi}{N}\right) & -\sin \left(\frac{N \cdot 2 \pi}{N}\right)
\end{array}\right\}} \\
& \text { and }\left\{\mathbf{Q}_{\mathbf{0}}\right\}_{2 N \times 1}=\left\{\begin{array}{c}
\mathrm{R}\left(Q_{\kappa_{1}}(\Delta x)\right) \\
\mathrm{I}\left(Q_{\kappa_{1}}(\Delta x)\right) \\
\mathrm{R}\left(Q_{\kappa_{2}}(\Delta x)\right) \\
\mathrm{I}\left(Q_{\kappa_{2}}(\Delta x)\right) \\
\ldots \\
\mathrm{R}\left(Q_{\kappa_{N}}(\Delta x)\right) \\
\mathrm{I}\left(Q_{\kappa_{N}}(\Delta x)\right)
\end{array}\right\}
\end{aligned}
$$

The result of Fourier transform is a symmetric spectrum. The terms in the second half of $\left\{\mathbf{Q}_{0}\right\}$ are conjugates of the first half ones. It is then possible to write a bijective 
matrix $[\mathbf{E}]_{N \times N}$ linking independently solutions in space domain $\{\mathbf{q}\}$ and in the Fourier domain $\{\mathbf{Q}\}$.

The general problem to solve in order to find periodic modes is

$$
[\mathbf{M}]_{N \times N}\{\ddot{\mathbf{q}}(\mathbf{t})\}_{N \times 1}+[\mathbf{K}]_{N \times N}\{\mathbf{q}(\mathbf{t})\}=0
$$

As global geometry of the track is periodic, dynamic stiffness of the structure is block diagonal and can be written as a function of the stiffness of the first slice $\left[\mathbf{Z}^{\mathbf{1}}\right]$ :

$$
[\mathbf{Z}]_{N \times N}=\left[\begin{array}{cccc}
{\left[\mathbf{Z}^{\mathbf{1}}\right]} & 0 & \cdots & 0 \\
0 & {\left[\mathbf{Z}^{\mathbf{1}}\right]} & \cdots & 0 \\
\cdots & \cdots & \cdots & \cdots \\
0 & 0 & \cdots & {\left[\mathbf{Z}^{\mathbf{1}}\right]}
\end{array}\right]
$$

At this point, damping matrix is not taken into account, it will be determined at the end of the reduction process. Dynamic stiffness matrix of the slice is then defined as $\left[\mathbf{Z}^{\mathbf{1}}\right]=\left[\mathbf{M}^{\mathbf{1}}\right]-\omega^{2}\left[\mathbf{K}^{\mathbf{1}}\right]$

The problem to solve, using Equation (5) is then $[\mathbf{E}]^{T}[\mathbf{Z}][\mathbf{E}]\left\{\mathbf{Q}_{\mathbf{0}}\right\}=\{\mathbf{F}\}$. As $[\mathbf{E}]^{T}[\mathbf{Z}][\mathbf{E}]$ is a block diagonal matrix, the resolution for the structure can be reduced to a resolution in the reference slice following:

$$
\left[\begin{array}{cc}
{\left[\mathbf{K}^{\mathbf{1}}\right]-\omega^{2}\left[\mathbf{M}^{\mathbf{1}}\right]} & 0 \\
0 & {\left[\mathbf{K}^{\mathbf{1}}\right]-\omega^{2}\left[\mathbf{M}^{\mathbf{1}}\right]}
\end{array}\right]\left\{\begin{array}{l}
\operatorname{Re}\left(Q_{\kappa_{n}}(\Delta x)\right) \\
\operatorname{Im}\left(Q_{\kappa_{n}}(\Delta x)\right)
\end{array}\right\}=\left\{\begin{array}{l}
\operatorname{Re}\left(F_{\kappa_{n}}\right) \\
\operatorname{Im}\left(F_{\kappa_{n}}\right)
\end{array}\right\}
$$

To compute static modes (corresponding to $\omega=0$ in Equation (8)), 5 values of wavenumber are chosen, evenly spaced in the $[0, \pi]$ interval. These values are labeled $\kappa_{s i}$, with $i=1$ to 5 ). For each one of these values the following equation is solved:

$$
\left[\begin{array}{cc}
{\left[\mathbf{K}^{\mathbf{1}}\right]} & 0 \\
0 & {\left[\mathbf{K}^{\mathbf{1}}\right]}
\end{array}\right]\left\{\begin{array}{l}
\operatorname{Re}\left(Q_{\kappa_{s i}}(\Delta x)\right. \\
\operatorname{Im}\left(Q_{\kappa_{s i}}(\Delta x)\right.
\end{array}\right\}=\left\{\begin{array}{l}
\operatorname{Re}\left(F_{\kappa_{s i}}\right) \\
\operatorname{Im}\left(F_{\kappa_{s i}}\right)
\end{array}\right\}
$$

The subspace linked to static deformation is then:

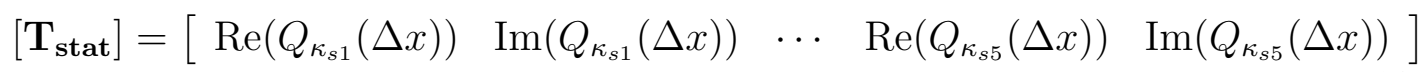

To build the subspace of periodic modes, an additional condition to take into account is the continuity of displacement at slices interfaces. To do so, for a given slice numbered n, two observation matrices are defined: $\left[\mathbf{c}_{1}^{\mathbf{n}}\right]$ for the DOFs of the left interface and $\left[\mathbf{c}_{\mathbf{r}}^{\mathbf{n}}\right]$ for the DOFs of the right interface. Following notations of Equation (1), they are defined as:

$$
\begin{aligned}
& \left\{\mathbf{q}_{1}^{\mathbf{n}}\right\}=\left[\mathbf{c}_{\mathbf{l}}^{\mathbf{n}}\right]\left\{\mathbf{q}^{\mathbf{s}}\right\} \\
& \left\{\mathbf{q}_{\mathbf{r}}^{\mathbf{n}}\right\}=\left[\mathbf{c}_{\mathbf{r}}^{\mathbf{n}}\right]\left\{\mathbf{q}^{\mathbf{s}}\right\}
\end{aligned}
$$

The continuity of displacement between adjacent slices can be expressed as $\left\{\mathbf{q}_{\mathbf{r}}^{\mathbf{n}}\right\}=$ $\left\{\mathbf{q}_{\mathbf{l}}^{\mathbf{n}+\mathbf{1}}\right\}$. If slices are identically meshed, then $\left[\mathbf{c}_{\mathbf{l}}\right]$ and $\left[\mathbf{c}_{\mathbf{r}}\right]$ are the same for all slices. 
Continuity condition can be expressed as (see [14] for more details):

$$
\left[\mathbf{c}_{\mathbf{r}}\right]\left\{\mathbf{Q}_{\mathbf{0}}\right\}=\left[\mathbf{c}_{\mathbf{l}}\right]\left\{\mathbf{Q}_{\mathbf{0}}\right\} \mathrm{e}^{\mathrm{i} \frac{2 \pi}{\mathrm{n}}}
$$

This equation is a periodicity of the second kind [15], and reflects the reproduction of harmonics after a wavelength $n \Delta x$. In Dynavoie, two periodicity lengths are considered: $n=5$ and $n=50$.

For each one of these values, the following equation is solved:

$$
\left[\begin{array}{cc}
{\left[\mathbf{K}^{\mathbf{1}}\right]-\omega^{2}\left[\mathbf{M}^{\mathbf{1}}\right]} & 0 \\
0 & {\left[\mathbf{K}^{\mathbf{1}}\right]-\omega^{2}\left[\mathbf{M}^{\mathbf{1}}\right]}
\end{array}\right]\left\{\begin{array}{l}
\operatorname{Re}\left(Q_{\kappa_{n}}(\Delta x)\right) \\
\operatorname{Im}\left(Q_{\kappa_{n}}(\Delta x)\right)
\end{array}\right\}=0
$$

A maximal frequency of interest $f_{\max }=100 \mathrm{~Hz}$ is defined, and only eigen modes verifying the condition $f<f_{\max }$ are selected to build the subspace, $n_{m p}$ is the number of modes satisfying this condition. This condition determines the areas of validity of the model.

The subspace of periodic modes is then:

$$
\left[\mathbf{T}_{\mathbf{d y n}}\right]=\left[\begin{array}{ll}
\operatorname{Re}\left(Q_{\kappa_{n}}(\Delta x)\right)_{N \times n_{m p}} & \operatorname{Im}\left(Q_{\kappa_{n}}(\Delta x)\right)_{N \times n_{m p}}
\end{array}\right]
$$

\subsection{Building of the basis}

At this stage, there is no reason for the subspace $\left[\mathbf{T}_{\mathbf{1}}\right]=\left[\begin{array}{ll}T_{\text {stat }} & T_{d y n}\end{array}\right]$ to be a basis. Some vectors of $\left[\mathbf{T}_{\mathbf{1}}\right]$ can be collinear. To transform $\left[\mathbf{T}_{\mathbf{1}}\right]$ in an orthogonal basis with respect to mass and stiffness matrices, the following conditions are specified:

1. $\left[\mathbf{T}_{\mathbf{1}}\right]^{T}[\mathbf{M}]\left[\mathbf{T}_{\mathbf{1}}\right]=[\mathbf{I}]$

2. $\left[\mathbf{T}_{\mathbf{1}}\right]^{T}[\mathbf{K}]\left[\mathbf{T}_{\mathbf{1}}\right]=\operatorname{diag}\left[\omega_{\mathbf{j}}^{\mathbf{2}}\right]$

Then, modeshapes linked to the deformation of the rail are added to $\left[\mathbf{T}_{\mathbf{1}}\right]$. This subspace is labelled $\left[\mathbf{T}_{\text {rail }}\right]$. The global reduction basis of the system is then:

$$
[\mathbf{T}]=\left[\begin{array}{ll}
T_{1} & T_{\text {rail }}
\end{array}\right]
$$

\subsection{Continuity of displacement}

The reduction basis $\left[\mathbf{T}_{\mathbf{1}}\right]$ describes the deformation of a slice without considering the continuity with adjacent slices. Deformation on the left and right interfaces of the slice can be different, so slices cannot be assembled directly to form the track. In order to add this continuity condition, the basis $\left[\mathbf{T}_{\mathbf{1}}\right]$ is decomposed as following:

$$
\left\{\begin{array}{c}
q_{l} \\
q_{c} \\
q_{\text {rail }} \\
q_{r}
\end{array}\right\}=\left[\begin{array}{llll}
T_{l} & T_{c} & T_{\text {rail }} & T_{r}
\end{array}\right]\left\{\begin{array}{c}
q_{l}^{R} \\
q_{c}^{R} \\
q_{\text {rail }}^{R} \\
q_{r}^{R}
\end{array}\right\}
$$




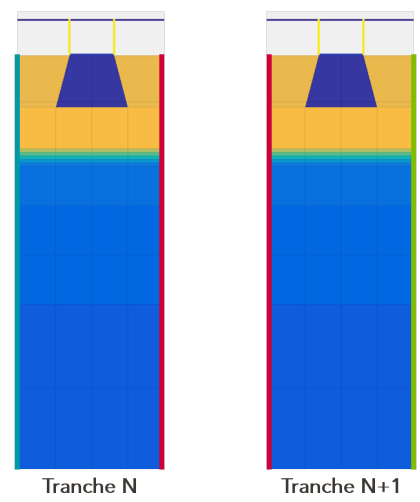

Figure 3: Visualisation of two slices of the track, numbered $N$ and $N+1$.

The following conditions are imposed:

- Non zero displacement on the left are generated with $T_{l}$. So $\left[\mathbf{c}_{\mathbf{l}}\right]\left[\begin{array}{lll}T_{c} & T_{\text {rail }} & T_{r}\end{array}\right]=$ 0

- Non zero displacement on the right are generated with $T_{r}$. So $\left[\mathbf{c}_{\mathbf{r}}\right]\left[\begin{array}{lll}T_{c} & T_{\text {rail }} & T_{r}\end{array}\right]=$ 0

- Traces equals on the left and on the right for $T_{l}$ and for $T_{r}:\left[\mathbf{c}_{\mathbf{l}}\right]\left[\mathbf{T}_{\mathbf{l}}\right]=\left[\mathbf{c}_{\mathbf{r}}\right]\left[\mathbf{T}_{\mathbf{r}}\right]$

- Then the last condition is direct continuity of displacement in the reduced subspace $\left\{\mathbf{q}_{\mathbf{r}}^{\mathbf{R N}}\right\}=\left\{\mathbf{q}_{\mathbf{l}}^{\mathbf{R}(\mathbf{N}+\mathbf{1})}\right\}$.

Various numerical strategies to build a basis following these four conditions exist and are not detailed here.

\subsection{Damping matrix}

Until this stage, no damping has been taken into account. The reduction base has been built taking into account only mass and stiffness matrices. The damping matrix $[C]$ is built in Dynavoie after the reduction stage. This matrix considers both hysteretic damping of each material (intern damping) and structural damping (damping due to interfaces), using a complex stiffness matrix $[\mathbf{K}]$ and loss factor for each element.

Then, the dynamic stiffness matrix can be written as follow

$$
[\mathbf{Z}(\mathbf{s})]=[\mathbf{K}]+s[\mathbf{C}]+s^{2}[\mathbf{M}]
$$

with $s=i w$. An estimation of the dynamic stiffness matrix is given by

$$
[\hat{\mathbf{Z}}(\mathbf{s})] \simeq[\mathbf{K}]+i\left[\mathbf{B}\left(\eta_{\mathbf{m}}\right)\right]+s^{2}[\mathbf{M}]
$$


where $[\mathbf{B}]$ is a hysteretic damping matrix that includes loss factors $\eta_{m}$ of each material:

$$
[\mathbf{B}]=\sum_{m} \eta_{m} K_{m}
$$

with $m$ representing each material of the model.

The dynamic stiffness is then

$$
[\hat{\mathbf{Z}}(\mathbf{s})]=\sum_{m}\left(\left(1+i \eta_{m}\right) \cdot\left[\mathbf{K}_{\mathbf{m}}\right]\right)+s^{2}[\mathbf{M}]
$$

The periodic modes $\left\{\boldsymbol{\Phi}_{\mathbf{j}}\right\}$ are solutions on a slice of

$$
\left(\left[\mathbf{K}_{\text {slice }}\right]-\omega_{j}^{2}\left[\mathbf{M}_{\text {slice }}\right]\right)\left\{\boldsymbol{\Phi}_{\mathbf{j}}\right\}=0
$$

Performing orthogonalization with mass and stiffness matrices leads to

$$
\begin{aligned}
{\left[\mathbf{\Phi}_{\mathbf{j}}\right]^{T}[\mathbf{M}]\left[\mathbf{\Phi}_{\mathbf{j}}\right] } & =[\mathbf{I}] \\
{\left[\mathbf{\Phi}_{\mathbf{j}}\right]^{T}[\mathbf{K}]\left[\mathbf{\Phi}_{\mathbf{j}}\right] } & =\operatorname{diag}\left[\omega^{\mathbf{2}}\right]
\end{aligned}
$$

Then, the viscous damping matrix of the system is $[\boldsymbol{\Gamma}]$ defined as

$$
[\boldsymbol{\Gamma}]=\left[\boldsymbol{\Phi}_{\mathbf{j}}\right]^{T}[\mathbf{C}]\left[\boldsymbol{\Phi}_{\mathbf{j}}\right]
$$

which leads for a unique slice to

$$
\left[\mathbf{\Phi}_{\mathbf{j}}\right]^{T}\left[\mathbf{Z}_{\text {slice }}\right]\left[\boldsymbol{\Phi}_{\mathbf{j}}\right]=s^{2}[\mathbf{I}]+s[\boldsymbol{\Gamma}]+\operatorname{diag}\left[\omega_{\mathbf{j}}^{\mathbf{2}}\right] .
$$

Combining Equation (17) and Equation (18), damping is given by $[\mathbf{B}] \simeq \omega[\mathbf{C}]$, which leads using (23) to

$$
\omega[\boldsymbol{\Gamma}] \simeq\left[\boldsymbol{\Phi}_{\mathbf{j}}\right]^{T}[\mathbf{B}]\left[\mathbf{\Phi}_{\mathbf{j}}\right]=\left[\mathbf{B}_{\mathbf{R}}\right]
$$

so

$$
\left[\boldsymbol{\Gamma}_{\mathbf{i j}}\right]=\left\{\frac{1}{\sqrt{\omega_{\mathbf{i}}}}\right\} \cdot\left[\mathbf{B}_{\mathbf{R}_{\mathbf{i j}}}\right] \cdot\left[\frac{1}{\sqrt{\omega_{\mathbf{j}}}}\right]
$$

For all $j$ :

- $\Gamma_{j j} \cdot \omega_{j R}=\Phi_{j R}^{T} \cdot B \cdot \Phi_{j R}$

- $\Gamma_{j j}=2 \cdot \xi_{j} \cdot \omega_{j}$

The damping coefficient $\xi_{j}$ for each mode $\left\{\boldsymbol{\Phi}_{\mathbf{j}}\right\}$ in the reduction base $[\mathbf{T}]$ can be given by

$$
\xi_{j}=\frac{1}{2 \omega_{j}^{2}} \Phi_{j}^{T}\left(\sum_{m} \eta_{m} K_{m}\right) \Phi_{j} .
$$

According to [16], with the orthogonality condition to the mass matrix 22.1 verified the following relations can be written: 


$$
\left([\mathbf{\Phi}]^{T}[\mathbf{M}]\right)[\mathbf{\Phi}]=[\mathbf{\Phi}]^{T}([\mathbf{M}][\mathbf{\Phi}])=[\mathbf{I}]=[\mathbf{\Phi}]\left([\mathbf{\Phi}]^{T}[\mathbf{M}]\right)=([\mathbf{M}][\mathbf{\Phi}])[\mathbf{\Phi}]^{T}
$$

Finally, by putting aside matrix $[\mathbf{C}]$ in Equation (23), and using $[\boldsymbol{\Phi}]^{-1}=[\boldsymbol{\Phi}]^{T}[\mathbf{M}]$ and $[\boldsymbol{\Phi}]^{T-1}=[\mathbf{M}][\boldsymbol{\Phi}]$ (Equation (28)), the viscous damping matrix on physical coordinates is expressed as

$$
[\mathbf{C}]=[\mathbf{M}][\mathbf{\Phi}][\boldsymbol{\Gamma}][\boldsymbol{\Phi}]^{T}[\mathbf{M}]
$$

\subsection{Building of the track}

For each individual slice, the problem to solve is given by

$$
\left[\mathbf{M}_{\mathbf{R}}\right]\left\{\ddot{\mathbf{q}}_{\mathbf{R}}(\mathbf{t})\right\}+\left[\mathbf{C}_{\mathbf{R}}\right]\left\{\dot{\mathbf{q}}_{\mathbf{R}}(\mathbf{t})\right\}+\left[\mathbf{K}_{\mathbf{R}}\right]\left\{\mathbf{q}_{\mathbf{R}}(\mathbf{t})\right\}=[\mathbf{T}]^{T}\{\mathbf{f}(\mathbf{t})\}
$$

with $\left[\mathbf{M}_{\mathbf{R}}\right],\left[\mathbf{C}_{\mathbf{R}}\right]$ and $\left[\mathbf{K}_{\mathbf{R}}\right]$ respectively the mass, damping and stiffness reduced matrices. These matrices are square, diagonals and their dimension is $N_{R} \times N_{R}$.

Dynavoie then assembles the slices to build the full 3D model

$$
\left[\begin{array}{cccc}
Z_{R} & 0 & \cdots & 0 \\
0 & Z_{R} & \cdots & 0 \\
\vdots & \vdots & \ddots & \vdots \\
0 & 0 & \cdots & Z_{R}
\end{array}\right]\left\{\begin{array}{c}
q_{R 1} \\
q_{R 2} \\
\vdots \\
q_{R N}
\end{array}\right\}=[\mathbf{T}]^{T}\{\mathbf{f}(\mathbf{x}(\mathbf{t}), \mathbf{t})\}
$$

with $\left[\mathbf{Z}_{\mathbf{R}}\right]=s^{2}\left[\mathbf{M}_{\mathbf{R}}\right]+s\left[\mathbf{C}_{\mathbf{R}}\right]+\left[\mathbf{K}_{\mathbf{R}}\right]$.

Equation (31) allows to compute reduced displacement $\left\{\mathbf{q}_{\mathbf{R i}}\right\}$ with $i \in[1, N]$. Then, with $\{\mathbf{q}\}=[\mathbf{T}]\left\{\mathbf{q}_{\mathbf{R}}\right\}$ the displacement field $\{\mathbf{q}\}$ on the physical DOFs can be computed.

\subsection{Numerical performance}

The final purpose of Dynavoie is to reduce time computation and storage compared to a full 3D FEM. This objective is achieved: Dynavoie divides by 100 the number of degrees of freedom of the model compared to a full 3D FEM. For example, to model $60 \mathrm{~m}$ of track with quadratic elements requires 4 million DOF in 3D full FE, and only 30000 with Dynavoie. Computing 11000 time steps in this configuration takes 30 minutes with Dynavoie, several days with a full 3D FEM.

\section{Test site and experimental results}

In this section, a transition zone, that is modeled using Dynavoie in section 4 , is presented and experimental results are briefly described. 


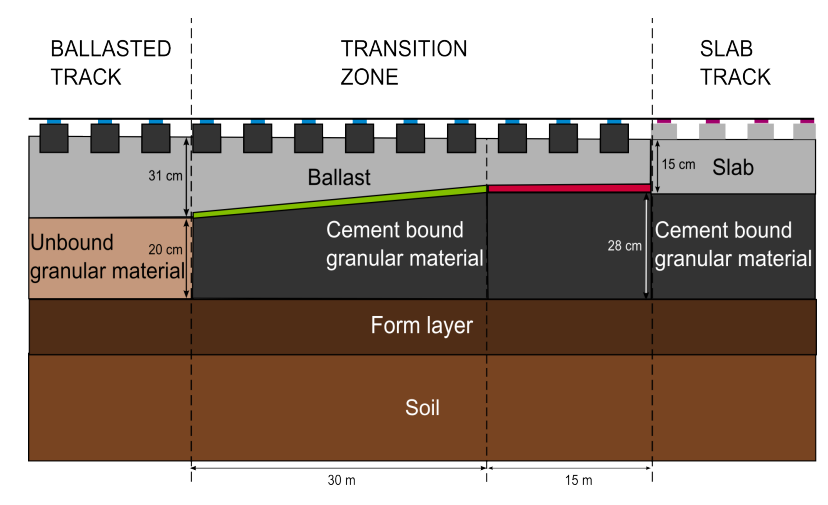

Figure 4: Description of Chauconin test site (adapted from [17]).

\subsection{Test site}

The chosen area is located in the French High Speed Line between Paris and Strasbourg and presents a transition between ballasted and slab track as displayed in Figure 4. Only the part with a ballast layer is studied in this work, but slab track could also be modeled with Dynavoie.

There are three different ballasted track designs in this zone:

- The ballasted track with properties of a conventional high speed design.

- The transition zone with mat 2 that provides the transition of geometry for the substructure between ballasted track and slab track. In the substructure, the compacted standard granular material layer is replaced by cement bound granular material. Between ballast and subgrade a polyurethane mat is introduced to provide the stiffness transition between slab track and ballasted track.

- The transition zone with mat 1 has the same characteristics as the first part of the transition, except for the ballast mat which is softer than in the previous area to progressively reach the slab track stiffness.

\subsection{Quasi-static measurements}

The first series of measurement in this area is EMW quasi-static rail deflection measurement [18]. This train belongs to CFF (Chemins de Fers Fédéraux Suisses) and measures axial deflection of the rail under a load of $20 \mathrm{t}$ at a speed of $15 \mathrm{~km} / \mathrm{h}$.

Stiffness measurements have been performed in 2008 and 2011 on this specific area. The results of measurements are presented in Figure 5 and average values by areas are summed up in Table 1.

Conclusions are the following:

- Despite local irregularities in the track deflection, amplitudes varies mainly when the substructure changes. In particular, deflection is twice as high in the transition zone than in the ballasted track. 

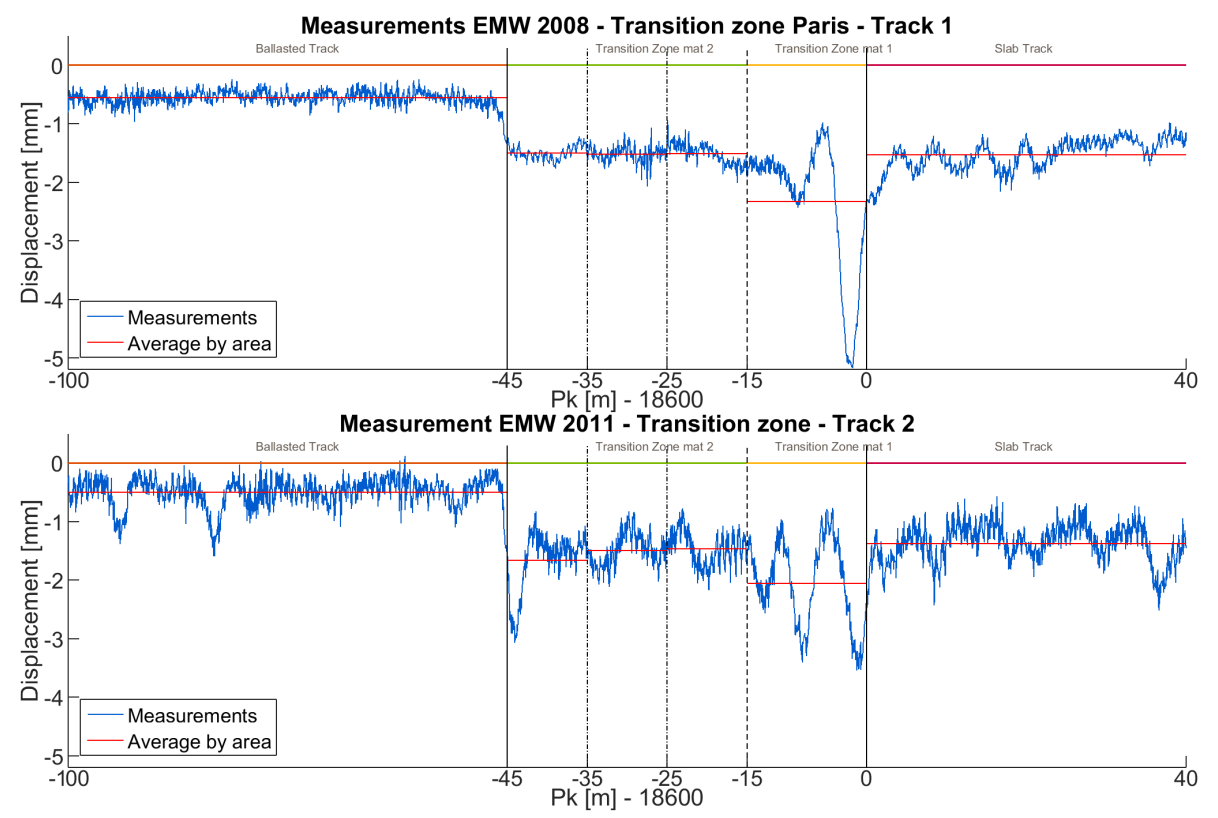

Figure 5: EMW measurements in the transition zone in 2008 and 2011.

\begin{tabular}{|c|c|c|c|c|}
\hline & Ballasted Track & TZ mat 2 & TZ mat 1 & Slab Track \\
\hline 2008 & -0.55 & -1.51 & -2.32 & -1.53 \\
\hline 2011 & -0.50 & -1.46 & -2.05 & -1.37 \\
\hline
\end{tabular}

Table 1: Mean values of quasi-static displacement under $20 \mathrm{t}$ measured by EMW in 2008 and in 2011

- The difference of properties between the two mats in the two areas of the transition is also visible: deflection is higher in zone with mat 1 than in zone with mat 2. This deflection change is due to the mat as it is the only difference in the track structures between the two areas.

- Much higher displacement is shown at the approach of the slab in 2008 and 2011, and between the ballasted track and the transition zone in 2011, which is characteristic of hanging sleepers that have been observed.

These measures reveal that static dimensioning of the track is not optimized as deflection change is not progressive from the ballasted track to the slab track. Hanging sleepers are due to ballast differential settlement [19] and according to [20], ballast settlement is mainly due to acceleration level on sleepers. A further step is to look at dynamic response of this transition zone.

\subsection{Dynamic measurements}

Two sets of dynamic measurements are performed in addition. The first are measurements of rail displacement under hammer impacts at the top of the rail. This gives 


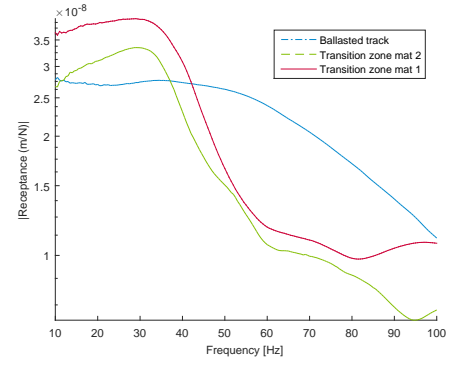

(a) Receptance

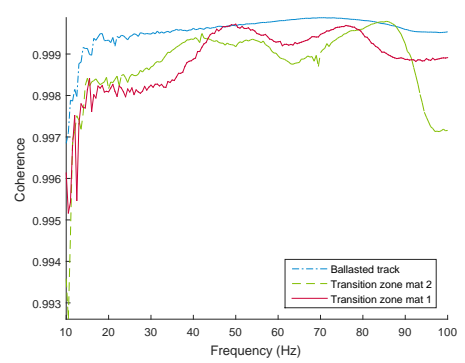

(b) Coherence

Figure 6: Direct receptance measured on ballasted track (in blue), on transition zone with mat 2 (green curve) and on transition zone with mat 1 (red curve) and corresponding coherence.

\begin{tabular}{|c|c|c|}
\hline & $\mathrm{L}[\mathrm{m}]$ & $\mathrm{f}[\mathrm{Hz}]$ \\
\hline Length of a car & 18.7 & 3.42 \\
\hline Distance between two boggies & 6.3 & 10.14 \\
\hline Distance between two axles & 3 & 21.29 \\
\hline Distance between sleepers & 0.6 & 106.48 \\
\hline
\end{tabular}

Table 2: Values of characteristic length for a speed of $230 \mathrm{~km} / \mathrm{h}$.

access to the transfer function (receptance) of the track in the frequency domain. The experimental setup is fully described in [21], only results are provided here in Figure 6.

The two curves corresponding to the transition zone are more flexible at low frequencies than the ballasted track. On the contrary, for frequencies higher than $40 \mathrm{~Hz}$, transition zone is stiffer than the ballasted track. The change of mat between the two curves of the transition zone is also clearly visible. In the previous part, the transition with mat 1 was shown more flexible than the one with mat 2 for quasi static measurements. Receptance test shows that this result is also valid for higher frequencies.

The second type of dynamic measures is acceleration of sleepers under the passing of trains. Commercial speed in this area is $230 \mathrm{~km} / \mathrm{h}$. This measuring campaign was fully described in [17]. Figure 7 shows power spectral density computed on 50 passages of simple unit TGVs.

Vertical lines in this Figure correspond to frequency linked to characteristic geometric lengths of the system, that are recalled in table 2, and their harmonics.

For the three zones, the peak of greatest amplitude is at $21 \mathrm{~Hz}$ and corresponds to distance between axles. Peaks corresponding to other characteristic lengths are also visible. Amplitude for the main peak is 3.5 times higher in transition zone with mat 2 than in the ballasted track, and 4.5 times higher in transition zone with mat 1 . For its first harmonic at $42 \mathrm{~Hz}$, this difference is even higher: amplitude is more than ten times superior in both areas of the transition than in the current ballasted track. On the contrary, the peak at $106 \mathrm{~Hz}$ linked to sleeper spacing gives birth to peaks with barely 

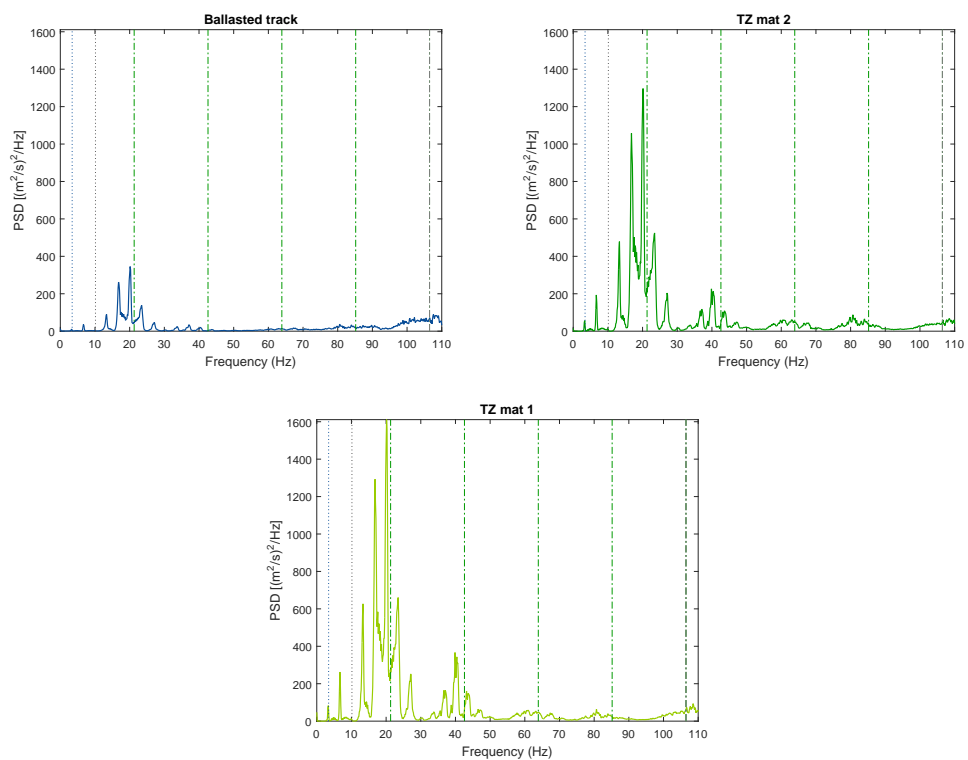

Figure 7: Power spectral density of acceleration measured on sleepers for a mean of 50 train passages.

the same amplitudes in every areas.

These observations have to be compared to the receptance measurement. According to [22], receptance shows the flexibility of the track. As below $50 \mathrm{~Hz}$, receptance curves are above the ballasted track. Then, it is not surprising that acceleration occurring below $50 \mathrm{~Hz}$ are amplified in the transition zone. To explain this difference between the structures, the transition zone is modeled with Dynavoie.

\section{Modeling of the transition}

All layers are considered as linear elastic with properties summarized in Table 3. Geometry is the one of Figure 4, and a section of the ballasted track is detailed in 8 .

Three different meshes are used to respectively model the ballasted track, the transition zone with mat 1 and the transition zone with mat 2.

To identify soil parameters, geological investigations performed before the line construction are used. These investigations revealed that the soil is composed of two layers: the first one of clay which is $3.8 \mathrm{~m}$ high above a limestone marl layer. To reduce boundary effects on soil, a gradient law for elastic modulus is considered:

$$
E=E_{0}\left(\frac{P_{\text {sup }}+\rho g z}{P_{0}}\right)^{n}
$$

The coefficient $n$ of Equation32 is set to 0.5, the target Young modulus value is 75 Mpa at $1 \mathrm{~m}$ depth in the first layer and a target value of $400 \mathrm{MPa}$ at $1 \mathrm{~m}$ of depth in the second and deepest soil layer are chosen. 
Table 3: Material properties

\begin{tabular}{ccccc} 
& $\begin{array}{c}E \\
(\mathrm{MPa})\end{array}$ & $\nu$ & $\begin{array}{c}\rho \\
\left(\mathrm{kg} / \mathrm{m}^{3}\right)\end{array}$ & $\eta$ \\
\hline Pad & 50 & 0.3 & 828 & 0.2 \\
Sleeper & 30000 & 0.25 & 2400 & 0 \\
Ballast & 200 & 0.3 & 1700 & 0.07 \\
Mat 1 & 0.59 & 0.3 & 900 & 0.1 \\
Mat 2 & 0.82 & 0.3 & 900 & 0.1 \\
Unbound granular material & 180 & 0.35 & 2135 & 0.04 \\
Cement bound granular material & 23000 & 0.25 & 2000 & 0.04 \\
Form layer & 200 & 0.3 & 1800 & 0.04 \\
Soil layer 1 & Gradient & 0.2 & 1800 & 0.2 \\
Soil layer 2 & Gradient & 0.2 & 1800 & 0.2
\end{tabular}

\section{Results}

The Figure 9 shows the compared receptance for the ballasted track and the transition zones. The combination of resonance followed by an antiresonance is a classical characteristic of isolation systems. In case of the transition zone with mats, the top layers (superstructure and ballast) are suspended above the mat. This suspension mode is found at $40 \mathrm{~Hz}$ and appears as a peak in the receptance. Above the suspension mode, the isolation starts to kick-in and the level drops below that of the nominal design around $50 \mathrm{~Hz}$. Around $60 \mathrm{~Hz}$, the flexibility of the platform no longer has a small effect and the isolation loses its efficiency leading to visible anti-resonance.

To confirm this analysis Figure 10 shows two cross-sections of displacement in the transition zone with mat. The response at $19.9 \mathrm{~Hz}$, before the resonance over the mat, shows in phase motion of ballast and soil. The line of zero response, shown in white, isolates an area at the edge of the track. At $50 \mathrm{~Hz}$, after the resonance, the line of zero response, occurs within the mat on the edge of the ballast and slightly below at the center of the track. Thus the expected phase opposition between ballast and soil motion is shown, which is characteristic of the isolation.

Now, calculation can be made in the time domain to see how the ballasted layer is influenced by this resonance. To do so, ballasted track and transition zone with mat 2 are modeled in one unique model. This implies considering two basic slices for the reduction part, and computing periodic and static deformation in both of them. Time computation is made for two moving loads, spaced by $3 \mathrm{~m}$ (to represent the spacing of axles in a boggie). Specific analysis is made on the ballast layer as ballast has shown premature degradation in this area. Acceleration field in this layer is presented on Figure 11 for three positions of the loads: on the ballasted track, on the transition zone and between the two of them.

The first conclusion from this figure is that acceleration is twice as important in the transition zone than in the ballasted track for the ballast layer. Another feature that 


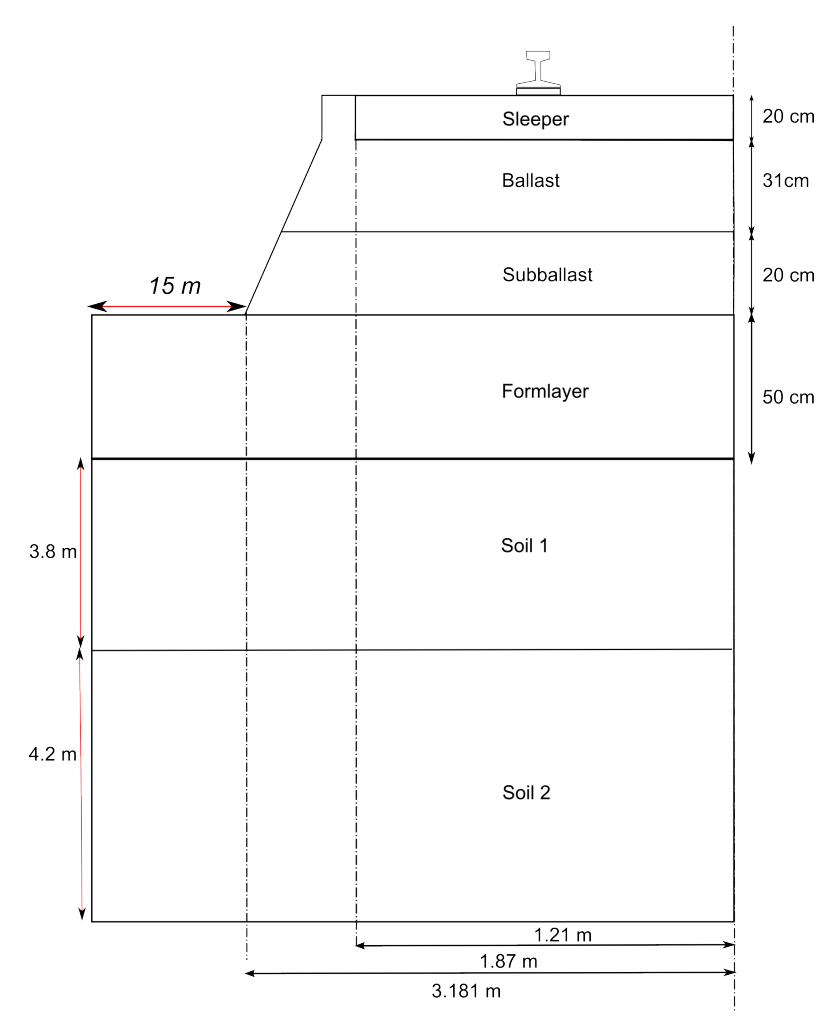

Figure 8: Section of the ballasted track

appears looking at these fields is that acceleration is more concentrated under sleepers in the transition zone. These two conclusions are elements that can explain uneven degradation of this layer while passing from ballasted track to transition zone, as well as in the transition zone itself.

\section{Conclusion}

A numerical tool, Dynavoie, able to model railway track, has been proposed and explained in this paper. Its final purpose is to quickly and correctly reproduce dynamic behavior of track and platform. The strategy lies on a periodic sub-structuring of the domain. Then, static and periodic modes representative of the track movement are computed and used to build a reduction basis. Using this basis allows to highly reduce the number of DOFs to consider, which reduces time computations and the memory required for models.

The case study presented is a transition zone between ballasted track and slab track on a French HSL. Experimental results show that viewed from the superstructure, both static and dynamic responses of the track are very different between ballasted track an the transition zone. Modeling the track reveals the existance of a resonance over the mat in the transition zone at $40 \mathrm{~Hz}$. In the time domain simulation, this resonance provokes higher accelerations in the ballast layer for the transition zone. 


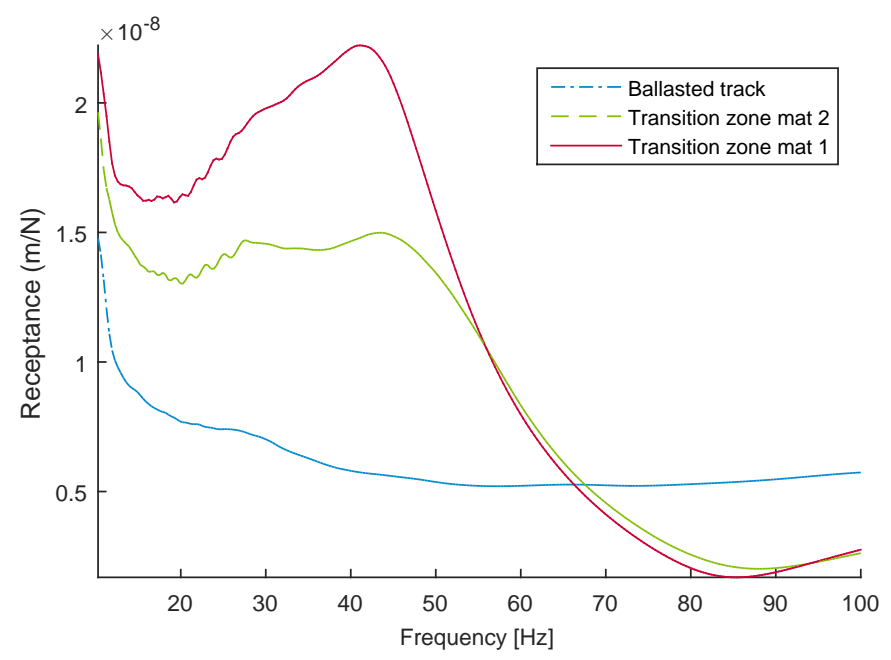

Figure 9: Computed receptance function for ballasted track (blue) and transition zone (in green with mat 2, in red with mat 1) in Chauconin test site.

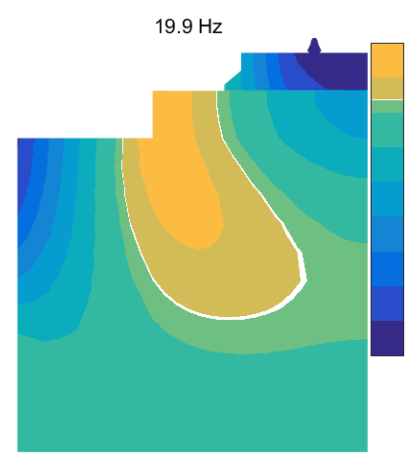

(a) TZ before resonance

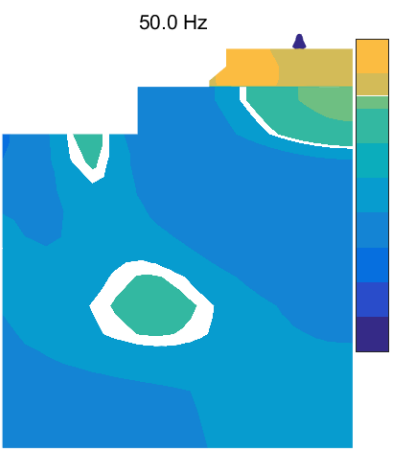

(b) TZ after resonance

Figure 10: Cross section of TZ displacement. White line corresponds to zero response.

\section{References}

[1] D. Ramirez Cardona, H. Di Benedetto, C. Sauzeat, Q. T. Nguyen, N. Calon, A. Robinet, Linear thermo-viscoelastic behaviour of bituminous mixtures used for railway trackbeds, in: J. Pombo (Ed.), Proceedings of the Second International Conference on Railway Technology: Research, Development and Maintenance, Civil-Comp Press, Stirlingshire, United Kingdom, 2014, paper 225.

[2] J. Semblat, A. Pecker, Waves and Vibrations in Soils: Earthquakes, Traffic, Shocks, Construction works, IUSS Press, 2009.

[3] J. Barbosa, P. Alves Costa, R. Calçada, Abatement of railway induced vibrations: Numerical comparison of trench solutions, Engineering Analysis with Boundary Elementsdoi:10.1016/j.enganabound.2014.11.029.

[4] M. Shahraki, C. Warnakulasooriya, K. J. Witt, Numerical Study of Transition Zone Between Ballasted and Ballastless Railway Track, Transportation Geotech- 


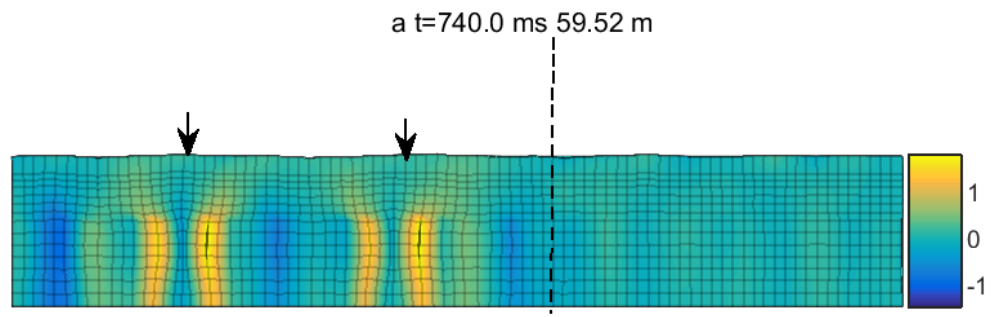

(a) Loads on the ballasted track

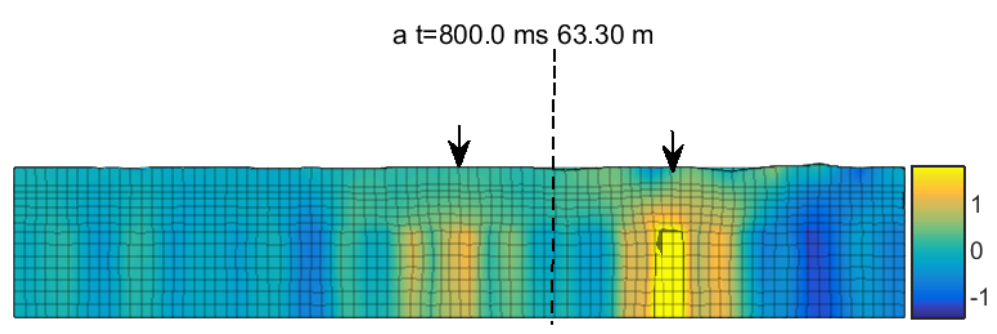

(b) One load on the ballasted track, another one on the transition zone

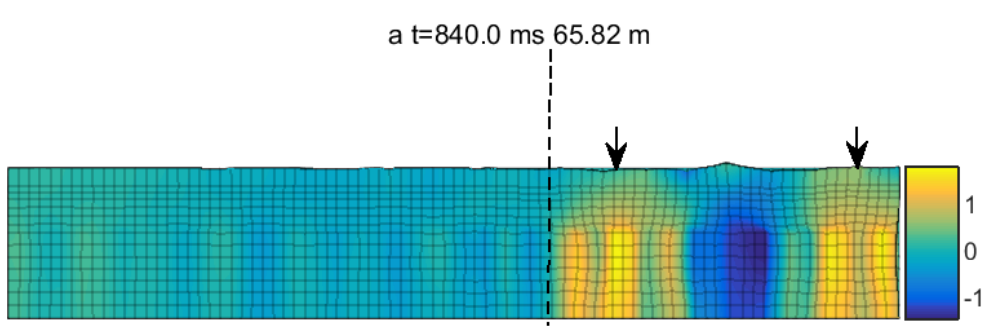

(c) Loads on the transition zone

Figure 11: Acceleration field on the ballast layer filtered between $1 \mathrm{~Hz}$ and $40 \mathrm{~Hz}$ 
nicsdoi:10.1016/j.trgeo.2015.05.001.

[5] S. François, M. Schevenels, P. Galvín, G. Lombaert, G. Degrande, A 2.5D coupled FE/BE methodology for the dynamic interaction between longitudinally invariant structures and a layered halfspace, Computer Methods in Applied Mechanics and Engineering 199 (23 - 24) (2010) 1536 - 1548. doi:http://dx.doi.org/10.1016/j.cma.2010.01.001.

[6] S. Ju, H. Li, Dynamic interaction analysis of trains moving on embankments during earthquakes, Journal of Sound and Vibration 330 (22) (2011) 5322 - 5332.

[7] N. Triepaischajonsak, D. Thompson, A hybrid modelling approach for predicting ground vibration from trains, Journal of Sound and Vibration

[8] G. Kouroussis, L. Van Parys, C. Conti, O. Verlinden, Using three-dimensional finite element analysis in time domain to model railway-induced ground vibrations, Advances in Engineering Software 70 (2014) 63-76.

[9] X. Bian, H. Jiang, C. Chang, J. Hu, Y. Chen, Track and ground vibrations generated by high-speed train running on ballastless railway with excitation of vertical track irregularities, Soil Dynamics and Earthquake Engineering

[10] H. Chebli, D. Clouteau, L. Schmitt, Dynamic response of high-speed ballasted railway tracks: 3D periodic model and in situ measurements, Soil Dynamics and Earthquake Engineering 28 (2) (2008) 118 - 131.

[11] L. Auersch, The excitation of ground vibration by rail traffic: theory of vehicletracksoil interaction and measurements on high-speed lines, Journal of Sound and Vibration 284 (1-2) (2005) 103-132.

[12] G. Lombaert, G. Degrande, Ground-borne vibration due to static and dynamic axle loads of InterCity and high-speed trains, Journal of Sound and Vibration 319 (3-5) (2009) 1036-1066.

[13] D. Connolly, P. Alves Costa, G. Kouroussis, P. Galvin, P. Woodward, O. Laghrouche, Large scale international testing of railway ground vibrations across Europe, Soil Dynamics and Earthquake Engineering 71 (2015) 1-12.

[14] E. Arlaud, S. C. D. Aguiar, E. Balmes, A numerical tool to assess the dynamic behaviour of different track designs, in: Railway Engineering, Edinburgh, 2015.

[15] D. Clouteau, M. Arnst, T. Al-Hussaini, G. Degrande, Freefield vibrations due to dynamic loading on a tunnel embedded in a stratified medium, Journal of Sound and Vibration 283 (1-2) (2005) 173-199.

[16] J. P. Bianchi, E. Balmes, G. Vermot des Roches, A. Bobillot, Using modal damping for full model transient analysis . Application to pantograph / catenary vibration ., in: Isma, 2010, pp. 1167-1180.

[17] S. Costa d'Aguiar, E. Arlaud, R. Potvin, E. Laurans, C. Funfschilling, Railway Transitional Zones: A case History from Ballasted to Ballastless Track, International Journal of Railway Technology 2 (3).

[18] G. Sodati, Emw kiesen 2006 : Gleiseinsenkungen mit dem einsenkungsmesswagen achslast $20 \mathrm{t}$, Tech. rep., SBB-report (01/2007).

[19] S. Zhang, X. Xiao, Z. Wen, X. Jin, Effect of unsupported sleepers on wheel/rail normal load, Soil Dynamics and Earthquake Engineering 28 (8) (2008) 662-673.

[20] A. Al Shaer, D. Duhamel, K. Sab, G. Foret, L. Schmitt, Experimental settlement 
and dynamic behavior of a portion of ballasted railway track under high speed trains, Journal of Sound and Vibration 316 (1-5) (2008) 211-233.

[21] E. Arlaud, S. C. D'Aguiar, E. Balmes, Dynamic behavior assessment of railway track in the frequency domain: numerical and experimental approaches, (Submitted to) Soil Dynamics and Earthquake Engineering - (-) - .

[22] A. P. D. Man, Dynatrack: A survey of dynamic railway track properties and their quality, Phd, Delft University (2002). 